\title{
Ice crystal characterization in cirrus clouds: a sun-tracking camera system and automated detection algorithm for halo displays
}

\author{
Linda Forster ${ }^{1}$, Meinhard Seefeldner ${ }^{1}$, Matthias Wiegner ${ }^{1}$, and Bernhard Mayer ${ }^{1,2}$ \\ ${ }^{1}$ Chair of Experimental Meteorology, Ludwig-Maximilians-Universität, Munich, Germany \\ ${ }^{2}$ Institut für Physik der Atmosphäre, Deutsches Zentrum für Luft- und Raumfahrt, Oberpfaffenhofen, Germany \\ Correspondence to: Linda Forster (linda.forster@physik.lmu.de)
}

Received: 20 January 2017 - Discussion started: 17 March 2017

Revised: 6 June 2017 - Accepted: 7 June 2017 - Published: 17 July 2017

\begin{abstract}
Halo displays in the sky contain valuable information about ice crystal shape and orientation: e.g., the $22^{\circ}$ halo is produced by randomly oriented hexagonal prisms while parhelia (sundogs) indicate oriented plates. HaloCam, a novel sun-tracking camera system for the automated observation of halo displays is presented. An initial visual evaluation of the frequency of halo displays for the ACCEPT (Analysis of the Composition of Clouds with Extended Polarization Techniques) field campaign from October to midNovember 2014 showed that sundogs were observed more often than $22^{\circ}$ halos. Thus, the majority of halo displays was produced by oriented ice crystals. During the campaign about $27 \%$ of the cirrus clouds produced $22^{\circ}$ halos, sundogs or upper tangent arcs. To evaluate the HaloCam observations collected from regular measurements in Munich between January 2014 and June 2016, an automated detection algorithm for $22^{\circ}$ halos was developed, which can be extended to other halo types as well. This algorithm detected $22^{\circ}$ halos about $2 \%$ of the time for this dataset. The frequency of cirrus clouds during this time period was estimated by colocated ceilometer measurements using temperature thresholds of the cloud base. About $25 \%$ of the detected cirrus clouds occurred together with a $22^{\circ}$ halo, which implies that these clouds contained a certain fraction of smooth, hexagonal ice crystals. HaloCam observations complemented by radiative transfer simulations and measurements of aerosol and cirrus cloud optical thickness (AOT and COT) provide a possibility to retrieve more detailed information about ice crystal roughness. This paper demonstrates the feasibility of a completely automated method to collect and evaluate a long-term database of halo observations and shows the potential to characterize ice crystal properties.
\end{abstract}

\section{Introduction}

Cirrus clouds represent about $30 \%$ of the global cloud coverage (Wylie et al., 1994) and play an important role in the earth's energy budget. They consist of small non-spherical ice crystals, which scatter and absorb solar radiation and emit thermal infrared radiation. Depending on which of the two effects dominates, cirrus clouds have either a cooling or a warming effect on climate. The radiative properties of cirrus clouds are governed not only by their optical thickness (COT) and ice crystal effective radius but also depend crucially on the ice crystal shape and orientation (Yi et al., 2013; Wendisch et al., 2007). Better knowledge of shape, surface roughness and orientation of ice crystals in cirrus clouds would therefore help to improve estimates of the radiative forcing of cirrus clouds as well as satellite retrievals of cirrus optical properties as discussed by Yang et al. (2015) and references therein.

Halo displays are produced by hexagonal ice crystals with smooth faces via refraction and reflection of sunlight. The formation of halo displays has already been described by Pernter and Exner (1910), Wegener (1925), Minnaert (1937) and by a number of later publications (Tricker, 1970; Greenler, 1980; Tape, 1994; Tape and Moilanen, 2006). One of the most common displays is the $22^{\circ}$ halo which appears as a bright ring around the sun at a scattering angle of about $22^{\circ}$ and is formed by randomly oriented hexagonal ice crystals. Further frequently observed halo displays are the parhelia of the $22^{\circ}$ halo, commonly called sundogs, which are caused by sunlight refracted by horizontally oriented hexagonal plates. Hexagonal ice crystal columns with their long axis oriented horizontally form another halo type: the upper and lower tan- 
(a)

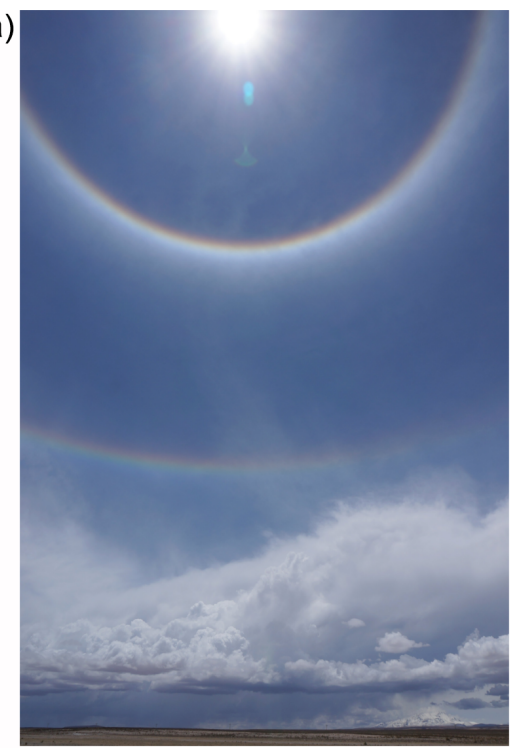

(b)

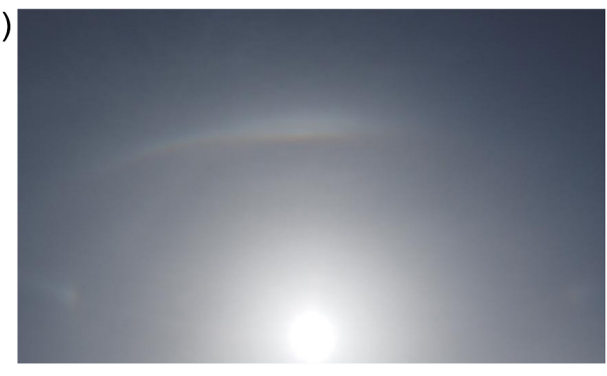

(c)

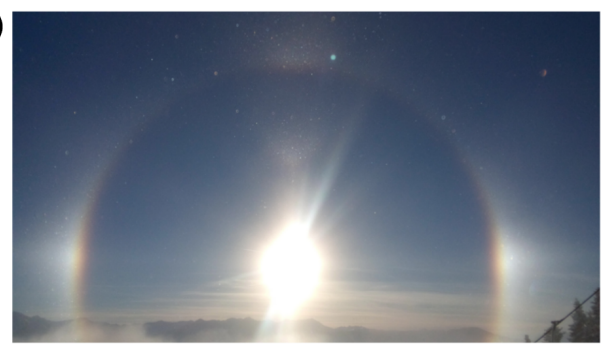

Figure 1. (a) A bright $22^{\circ}$ halo or circumscribed halo with infralateral arc below, Salar de Uyuni, Bolivia, 2 October 2014 (photograph by Leonhard Scheck). (b) Upper tangent arc with faint sundogs in Munich, Germany, 1 April 2014. The halo displays are faint due to the high aerosol concentration in the air. (c) A $22^{\circ}$ halo with upper tangent arc and bright sundogs on Mt. Hohe Salve, Austria, 18 January 2016 (photograph by Volker Freudenthaler).

gent arcs. Their shape changes with the solar elevation. When the sun is close to the zenith, both the upper and lower tangent arcs merge to the circumscribed halo. Figure 1 shows examples of the most frequent halo displays. The left image depicts a bright $22^{\circ}$ or circumscribed halo with a rare infralateral arc below. A faint upper tangent arc and two faint sundogs are shown on the upper right image, and very bright sundogs with a faint $22^{\circ}$ halo and a small upper tangent arc are displayed on the lower right image. Halos are not only beautiful optical displays but also contain valuable information about ice particle shape and orientation. Recent publications showed that the brightness contrast of the $22^{\circ}$ halo in ice crystal scattering phase functions is related to the aspect ratio and surface roughness of the crystals (van Diedenhoven, 2014). Quantitative analysis of, for example, the frequency of occurrence or brightness contrast of halo displays can therefore help to determine ice crystal properties, such as shape, surface roughness and orientation in cirrus clouds.

Probably the first reported photometric measurements of halo displays were performed by Lynch and Schwartz (1985), who took a photo of a $22^{\circ}$ halo around the moon with a Kodak Plus-X pan film camera. After digitizing the photo, the halo brightness and width was analyzed and compared with theoretical values to infer information about ice crystal size and shape.

In order to exploit the information content of halo displays, continuous long-term observations of cirrus clouds are required. In the 1990s many observations were collected by amateur halo-observing networks (Pekkola, 1991; Verschure, 1998), which is work-intensive and requires a lot of personnel. The largest dataset of halo observations has been collected by the German Arbeitskreis Meteore e.V. Sektion Halobeobachtungen (AKM, https://www.meteoros.de). The community was founded in 1990 and consists of a network of about 80 volunteers who collect halo observations on a monthly basis throughout Germany, Austria, Romania and the UK. Since 1986 more than 150000 observations of halo displays have been reported. The AKM collects information about the halo type and its duration, the type of cloud producing the halo, the weather situation during the observation (frontal system, precipitation) and more. These observations are valuable for obtaining an average frequency of the different halo displays in Europe. However, for a systematic comparison with other measurement data, continuous observations at a specific location for a long period of time are required.

An extensive long-term observation study of high-level clouds and halo displays was performed by Sassen et al. (2003), who evaluated a $\sim 10$-year record of photographic halo observations together with measurements with a polarization lidar and other remote sensing instruments at the Facility for Atmospheric Remote Sensing (FARS) in Salt Lake City, Utah. This study is also based on visually collected halo observations. A fisheye camera, which took pictures every $20 \mathrm{~min}$, was used in this study in combination with field notes and extra photographs to monitor optical displays. Sassen et al. (2003) pointed out that their optical display statistics are representative only for the observation area at FARS and that a common format for reporting atmospheric optical displays is needed to allow comparison of data from different 
locations. In order to perform long-term halo and cirrus observations, an automated low-maintenance system is needed which can be easily deployed at different locations.

We present the novel camera system HaloCam, designed for the automated observation of halo displays with high temporal and spatial resolution. Combined with a halo detection algorithm, HaloCam is, to our knowledge, the first fully automated camera system which can provide consistent longterm observations of halo displays. By evaluating the frequency of occurrence of halo displays and the fraction of cirrus clouds, the observations can contribute to gain more information about the dominating ice crystal properties.

The first section of this paper describes the setup and design of HaloCam. A first visual evaluation of the frequency of different halo displays using HaloCam observations is presented in Sect. 2.1. The following section explains the characterization and geometric calibration of HaloCam which is necessary for image processing and feature extraction of the halo displays. In the next section an automated halo detection algorithm based on a random forest classifier is presented and its implementation is described. Section 3.2 provides the results of the halo detection algorithm applied to HaloCam observations. Finally, the results of the halo display statistics are discussed with the help of radiative transfer simulations.

\section{The automated halo observation camera HaloCam}

In order to automatically collect halo observations, the suntracking camera system HaloCam was developed at the Meteorological Institute (MIM) of the Ludwig-MaximiliansUniversität (LMU), Munich, and installed on the rooftop platform as shown in Fig. 2. HaloCam consists of a weatherproof wide-angle camera and is mounted on a sun-tracking system. Using a sun-tracking mount is very suitable for the observation of halo displays and later image processing since it allows the alignment of the center of the camera with the sun. This implies that the recorded halo displays are also centered on the camera pictures. With this setup a small fixed shade is sufficient to protect the camera lens from direct solar radiation and to avoid overexposed pixels and stray light. The mount features two stepping motors with gear boxes for adjusting the azimuth and elevation angles of the camera position as described in Seefeldner et al. (2004) with an incremental positioning of 2.16 arcmin per step. The positioning of the mount is performed by passively tracking the sun: an algorithm calculates the current position of the sun, which is converted to incremental motor steps and moves the two motors accordingly. The pointing accuracy of the mount can be roughly estimated to about $\pm 0.5^{\circ}$ ( $2 \sigma$ standard deviation), which will be explained in more detail in Sect. 2.3. The camera (Mobotix S14D) is a light-weight modular system with an RGB CMOS sensor of $1 / 2^{\prime \prime}$ size. Combined with a lens of $22 \mathrm{~mm}$ focal length, it provides a horizontal and vertical field of view (FOV) of 90 and $67^{\circ}$, respectively. Further specifica-

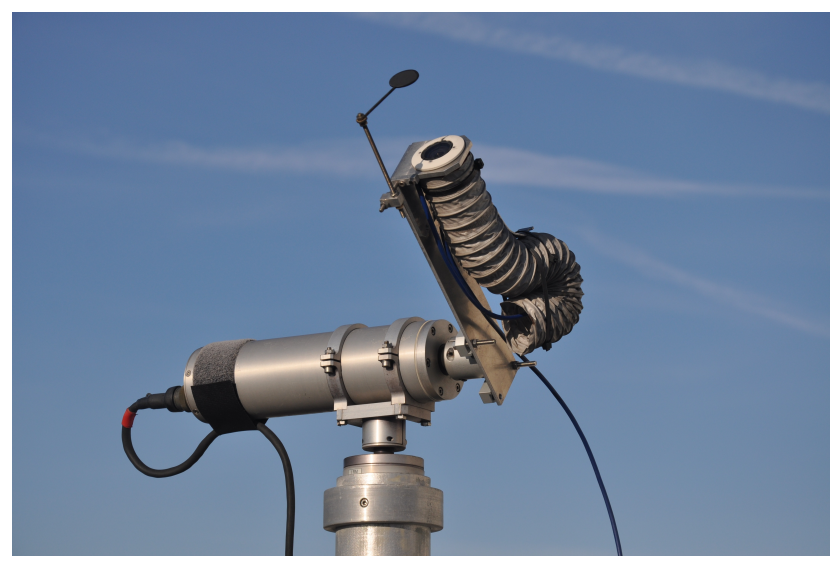

Figure 2. HaloCam: wide-angle camera (Mobotix S14D) with circular shade on a sun-tracking mount. The mount consists of two axes with stepping motors to adjust azimuth and elevation of the camera.

Table 1. HaloCam camera specifications.

\begin{tabular}{ll}
\hline Lens & \\
\hline Equivalent $35 \mathrm{~mm}$ focal length & $22 \mathrm{~mm}$ \\
Nominal focal length & $4 \mathrm{~mm}$ \\
Horizontal field of view & $90^{\circ}$ \\
Vertical field of view & $67^{\circ}$ \\
\hline Camera (Mobotix S14D flexmount) & \\
\hline Protection class & IP65, -30 to $+60^{\circ} \mathrm{C}$ \\
Sensor & $1 / 2^{\prime \prime}$ CMOS, RGB \\
& progressive scan \\
Sensor resolution & 3 MP \\
Compression formats & JPEG, MxPEG, M-JPEG \\
\hline
\end{tabular}

tions of the Mobotix S14D camera are listed in Table 1. The camera is operated in an automatic exposure mode and the image region used to determine the optimum exposure time is confined to the region where the $22^{\circ}$ halo occurs. This ensures that the pixels around the $22^{\circ}$ halo are not saturated. The camera FOV and the sensor resolution were chosen to optimize the trade-off between a large coverage of the sky with high spatial resolution and low image distortion. HaloCam allows for the observation of the $22^{\circ}$ halo, sundogs, and upper/lower tangent arcs or circumscribed halo, which are the most frequent halo displays according to Sassen et al. (2003) and the results of the AKM.

The HaloCam observations aim at gaining a better understanding of the relationship between halo displays and typical ice crystal properties in cirrus clouds. Hence, the observations can be limited to the most frequent halo displays without loosing relevant information about ice crystal shape and orientation while achieving a high spatial and temporal resolution of the scene. Every $10 \mathrm{~s}$, HaloCam's position relative to the sun is updated and a picture is recorded. HaloCam was 
installed in September 2013 on the rooftop platform of MIM (LMU) in Munich, where operational measurements are performed by a MIRA-35 cloud radar (Görsdorf et al., 2015), a CHM15kx ceilometer (Wiegner et al., 2014) and a sun photometer, which is part of the AERONET (Aerosol Robotic Network) network (Holben et al., 1998), as well as with the institute's own sun photometer SSARA (Sun-Sky Automatic Radiometer) (Toledano et al., 2009, 2011). HaloCam observations ideally complement these measurements to retrieve more detailed information about ice crystal properties.

\subsection{HaloCam observations - a first statistical evaluation}

HaloCam has been operated in Munich (Germany) since September 2013, where it provides continuous measurements including contributions to the ML-CIRRUS campaign in March and April 2014 (Voigt et al., 2017). It was installed in Cabauw (the Netherlands) only during the ACCEPT campaign (Analysis of the Composition of Clouds with Extended Polarization Techniques, Myagkov et al., 2016) in October and November 2014. A first visual evaluation of halo display frequency during ACCEPT (10 October until 14 November 2014) was performed. The results are displayed in Fig. 3 as a Venn diagram (Venn, 1880). The occurrence of each different halo type is visualized by a circle. The radius of each circle scales with the total observation time for the respective halo type. Cross sections between the circles indicate instances where two or three halo displays were visible at the same time. The observation time is given in hours. The total time of HaloCam observations, which were collected during daytime only, amounts to about $344 \mathrm{~h}$. With about $30 \mathrm{~h}$, halo displays were observed in almost $9 \%$ of the time. The presence of cirrus clouds within the HaloCam field of view was evaluated visually and amounts to about $110 \mathrm{~h}$. Thus, about $27 \%$ of the cirrus clouds produced a visible halo display. The $22^{\circ}$ halo (complete or partial) occurred in $16.2 \%$, the sundogs in $19 \%$ and the upper tangent arcs in $7.8 \%$ of the time when cirrus clouds were present. Circumscribed halos were not observed during the campaign due to the low solar elevations.

As illustrated in Fig. 3, sundogs were observed more often than $22^{\circ}$ halos, for about 21 vs. $18 \mathrm{~h}$. Thus, sundogs occurred in $70 \%$ and $22^{\circ}$ halos in $60 \%$ of the total halo observation time $(30 \mathrm{~h})$. Upper tangent arcs occurred in total for about $9 \mathrm{~h}$ $(30 \%)$ and were accompanied most of the time by $22^{\circ}$ halos and sundogs. Thus, the majority of the halo displays were produced by oriented ice crystals.

Compared to the findings of Sassen et al. (2003), the relative fraction of $22^{\circ}$ halos is roughly similar with $50 \%$, but sundogs with $12 \%$ and upper/lower tangent arcs with about $15 \%$ were far less frequent than observed during ACCEPT. The AKM observed the left and right sundogs with a relative frequency of $18 \%$ each, compared to $36 \%$ for the $22^{\circ}$ halos. Although the frequency of simultaneous occurrence of the

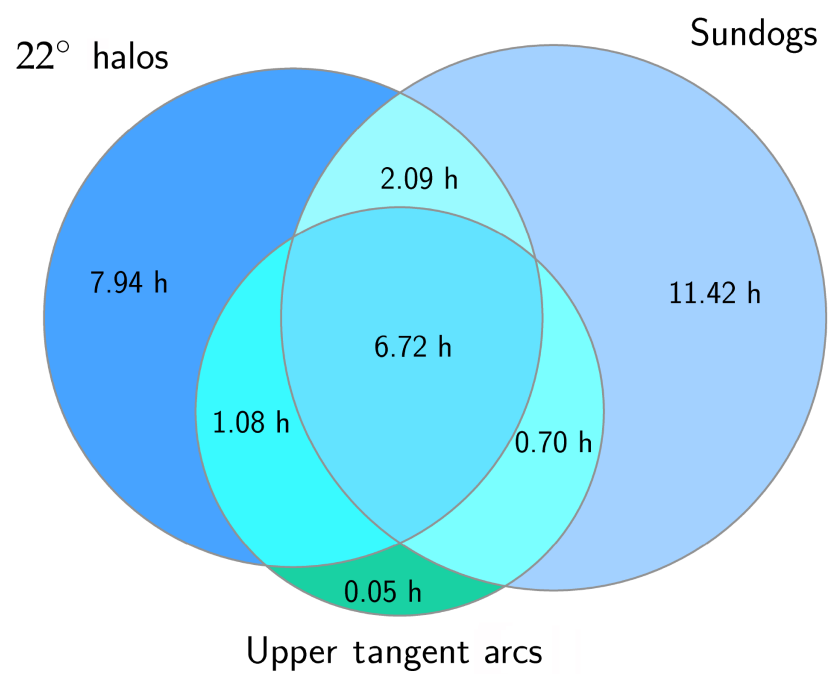

Figure 3. Halo display statistics from HaloCam observations during the ACCEPT campaign 10 October-14 November 2014. The observation times of $22^{\circ}$ halo, sundogs and upper tangent arc are provided in hours and are represented by the radii of the three circles. Cross sections of circles indicate time periods when two or three halo displays were visible simultaneously. The total observation time amounts to $344 \mathrm{~h}$.

left and right sundog is unknown (from the AKM database), one can deduce that the relative frequency of sundogs is at least $18 \%$ and thus larger than the result of Sassen et al. (2003). The reasons for the differences in the observed halo frequencies could be manifold: one main reason might be that a statistical evaluation over 6 weeks is compared to a database of 10 (Sassen et al., 2003) and 30 years (AKM). It is possible that the observation time during ACCEPT was not long enough to yield representative results for the frequency of the different halo displays. Another factor could be the observation site. The mountains in the east of Salt Lake City, the observation site of Sassen et al. (2003), could obscure the sun during periods with low solar elevation which are favorable for the formation of sundogs. So it is possible that on average fewer sundogs could have been observed in Salt Lake City than in Cabauw, which is surrounded by a rather flat landscape. Additionally, differences in the dominating weather patterns forming cirrus clouds in Salt Lake City and Cabauw could have an impact on halo formation as discussed in Sassen et al. (2003). For the AKM and the HaloCam dataset, information about dominating weather patterns for different halo displays is not available. Furthermore, the observation period during the ACCEPT campaign from October until mid-November was dominated by low solar elevations, which implies a higher chance for observing sundogs. Long-term observations have to be evaluated to obtain representative results of the frequency of the different halo types. To evaluate the large HaloCam dataset that has been collected for more than 2.5 years, an automated algorithm was developed for the detection of $22^{\circ}$ halos. The following sections 
(a)

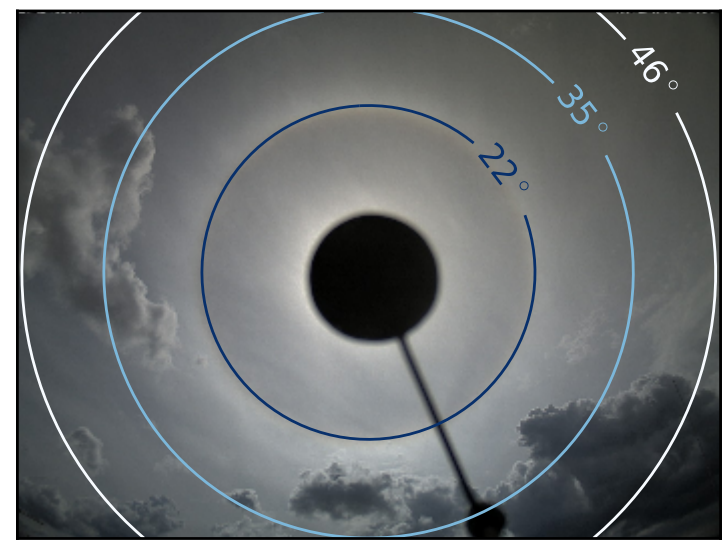

(b)

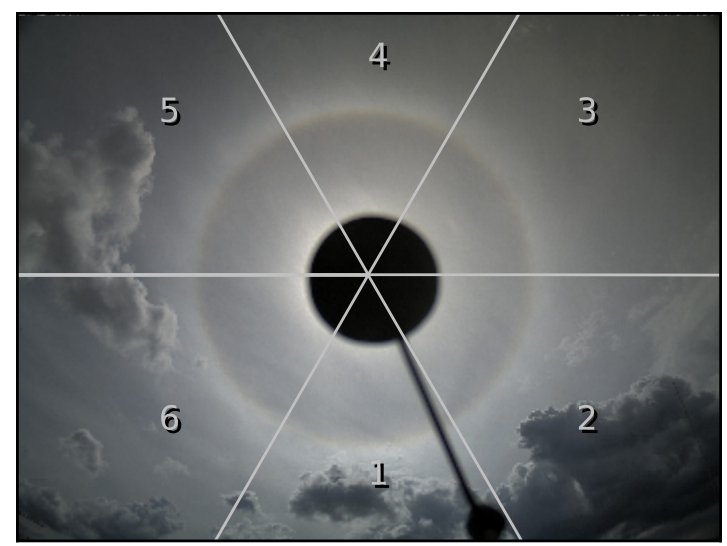

Figure 4. (a) HaloCam image from 12 May 2014, 13:52 UTC, with corresponding scattering angle $(\vartheta)$ grid and representative contour lines at 22,35 and $46^{\circ}$, (b) shows the relative azimuth $(\varphi)$ grid with numbered labels for the six image segments.

describe how the HaloCam images are processed and which features are extracted for an automated halo detection.

\subsection{Camera characterization and calibration}

Halo displays are single scattering phenomena and thus are directly linked to the optical properties of the ice crystals producing them. The ice crystal phase function predicts the scattering angle $\Theta$ of the $22^{\circ}$ halo relative to the sun. Thus, the analysis of the HaloCam images can be simplified significantly by mapping the image pixels to scattering angles. This means the camera has to be calibrated in order to determine the parameters for mapping the camera pixels to the real world spherical coordinate system. For this mapping the intrinsic camera parameters have to be determined, which are the focal lengths $\left(f_{x}, f_{y}\right)$ and image center coordinates $\left(c_{x}\right.$, $c_{y}$ ), as well as the distortion coefficients of the camera lens.

Different methods exist for the geometric calibration. Here, we use the method described by Zhang (2000), which is based on Heikkila and Silven (1997), to estimate the intrinsic camera parameters as well as the radial and tangential

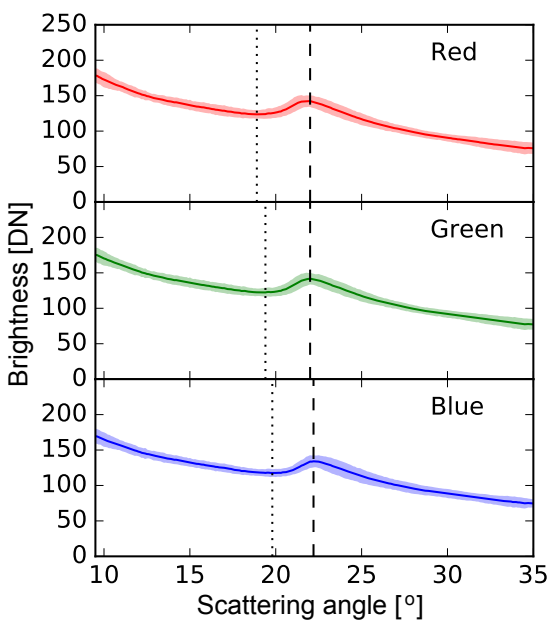

Figure 5. HaloCam image processing demonstrated for the measurements shown in Fig. 4, segment no. 4. The three panels show the brightness distributions (in digital numbers, DN) for the red, green and blue image channel as a function of the scattering angle. The solid line represents the brightness averaged azimuthally over the image segment, whereas the shading indicates the $2 \sigma$ standard deviation. The vertical lines pinpoint the scattering angles of the $22^{\circ}$ halo minimum (dotted) and maximum (dashed) for the RGB channels.

distortion parameters of the lens. This method requires several pictures of a planar pattern, for example, a chessboard pattern with known dimensions, taken with different orientations. The calibration method using a chessboard pattern was implemented in OpenCV by Itseez (2015) and is described in detail by Bradski and Kaehler (2008). Using the distortion coefficients and intrinsic parameters, the camera pixels can be undistorted and mapped to the world coordinate system. Thereby a zenith $(\vartheta)$ and azimuth angle $(\varphi)$ relative to the image center can be assigned to each pixel. Since the image center is pointing to the center of the sun, the relative zenith angle $(\vartheta)$ corresponds to the scattering angle $\Theta$ in this case.

An overlay of the scattering angle grid onto a HaloCam picture is shown in Fig. 4a with representative contour lines at $\vartheta=22,35$ and $46^{\circ}$. From the scattering angle grid the horizontal and vertical FOV can be calculated to $\sim 93.4$ and $\sim 70.2^{\circ}$, respectively. HaloCam images are recorded with a resolution of $1280 \times 960$ quadratic pixels, which results in an angular resolution of $\sim 0.07^{\circ}$ for both the horizontal and the vertical direction. Figure $4 \mathrm{~b}$ shows the relative azimuth angle grid, which is chosen such that the image is separated into six segments. For further analysis and feature extraction, each of these segments is averaged azimuthally.

\subsection{HaloCam image processing and feature extraction}

For processing, the HaloCam images can be decomposed into their red, green and blue color channels. The brightness $I$ of each pixel, provided in digital numbers (DN), can then 
Table 2. The $22^{\circ}$ halo features for the example of 12 May 2014 13:52 UTC (as in Fig. 5). The relative zenith angle (which corresponds to the scattering angle) is listed for the minimum $\vartheta_{\text {halo, min }}$ and maximum $\vartheta_{\text {halo, max }}$ brightness of the $22^{\circ}$ halo together with the brightness contrast, i.e., the halo ratio (HR) for the red, green and blue image channel.

\begin{tabular}{llll}
\hline & $\vartheta_{\text {halo, } \min }$ & $\vartheta_{\text {halo, } \max }$ & HR \\
\hline Red & $18.9^{\circ}$ & $22.0^{\circ}$ & 1.15 \\
Green & $19.4^{\circ}$ & $22.0^{\circ}$ & 1.16 \\
Blue & $19.8^{\circ}$ & $22.2^{\circ}$ & 1.14 \\
\hline
\end{tabular}

be represented as a data array with $1280 \times 960$ elements. As an example the HaloCam image of Fig. 4 is used to demonstrate how the images are processed in case of a $22^{\circ}$ halo. Figure 5 depicts the brightness distributions of the red, green and blue channel as a function of the scattering angle, averaged azimuthally over the uppermost image segment (no. 4 in Fig. 4b). The shaded areas around the lines in Fig. 5 represent twice the standard deviation of the averaged image region.

For analyzing the HaloCam observations several features can be extracted from the brightness distribution across the $22^{\circ}$ halo, which will be explained in the following. The angular position of the $22^{\circ}$ halo maximum $\left(\vartheta_{\text {halo, max }}\right)$ is found by searching for the maximum brightness in the interval $\left(21.0^{\circ}, 23.5^{\circ}\right)$. Then the angular position of the halo minimum $\left(\vartheta_{\text {halo, min }}\right)$ is determined by looking for the minimum brightness in the interval $\left(18.0^{\circ}, \vartheta_{\text {halo, max }}\right)$. Another important feature is the brightness contrast of the halo. In previous publications (Gayet et al., 2011; Shcherbakov, 2013; van Diedenhoven, 2014) the so-called "halo ratio" (HR) was introduced as a measure for the brightness contrast of the $22^{\circ}$ and $46^{\circ}$ halo in the scattering phase function. In analogy, here, the halo ratio is defined as the brightness $I$ at the scattering angle of the halo maximum $\vartheta_{\text {halo, max }}$ divided by the brightness at the scattering angle of the minimum $\vartheta_{\text {halo, min }}$ :

$\operatorname{HR}=I\left(\vartheta_{\text {halo, } \max }\right) / I\left(\vartheta_{\text {halo, } \min }\right)$.

As an example, the values for $I\left(\vartheta_{\text {halo, max }}\right)$ and $I\left(\vartheta_{\text {halo, min }}\right)$ are displayed in Fig. 7 by the blue triangles pointing up $(\max )$ and down $(\min )$, respectively. For clearsky conditions and homogeneous cloud cover, the brightness distribution decreases from the sun towards larger scattering angles, as shown in the example in Figs. 5 and 7. If $\mathrm{HR}<1$ the brightness at the scattering angle of the halo maximum $\left(I\left(\vartheta_{\text {halo, max }}\right)\right)$ is smaller than for the minimum $\left(I\left(\vartheta_{\text {halo, } \min }\right)\right)$, which is representative for a monotonically decreasing, featureless curve in this scattering angle region. This is the case for clear-sky conditions or homogeneous cloud cover without a halo. For HR $=1$ the brightnesses at the halo maximum and minimum are the same, causing a slight plateau in the brightness distribution. A distinct halo peak occurs for the condi-

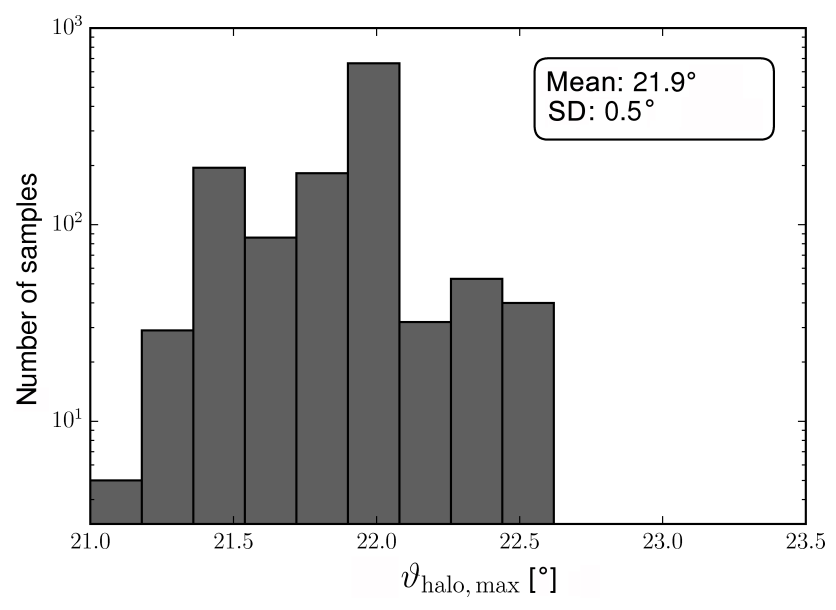

Figure 6. Distribution of the scattering angles of the $22^{\circ}$ halo brightness maximum $\vartheta_{\text {halo, max }}$ in degrees for 1289 randomly chosen and visually classified images using the uppermost image segment (no. 4). The mean value amounts to $21.9^{\circ}$ with a $2 \sigma$ confidence interval of $\pm 0.5^{\circ}$. Note the logarithmic scale of the $y$ axis.

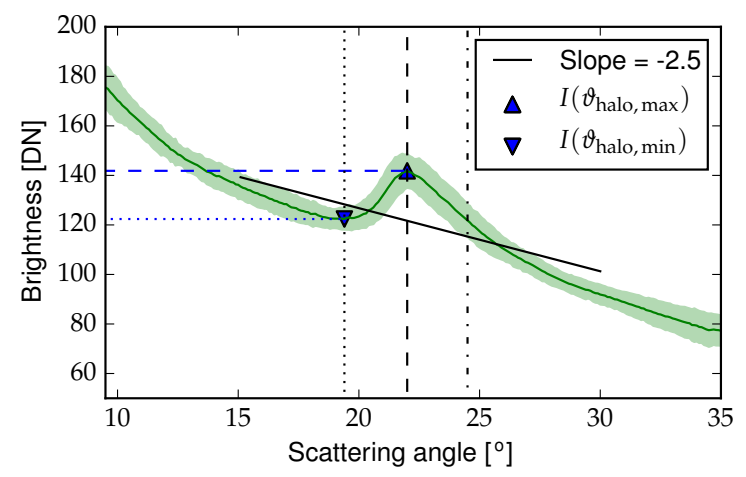

Figure 7. As Fig. 5, showing the first minimum (dotted) and the maximum (dashed) of the $22^{\circ}$ halo for the green channel. In addition, $\vartheta_{\text {halo, end }}$ is indicated (dash-dotted line), which represents the scattering angle of the same brightness as $\vartheta_{\text {halo, min }}$ and confines the halo peak. In this example $\vartheta_{\text {halo, end }}$ is located at about $24.5^{\circ}$. The corresponding brightness values $I\left(\vartheta_{\text {halo, min }}\right)$ and $I\left(\vartheta_{\text {halo, max }}\right)$ used to calculate the HR are marked with blue triangles pointing down (min) and up (max). The regression line of the averaged brightness distribution (solid black), which is evaluated between scattering angles of 15 and $30^{\circ}$, has a slope of -2.5 for this example.

tion HR $>1$. Thus, we assume HR $=1$ as lower threshold for the visibility of a halo.

For the example of Fig. 5 the $22^{\circ}$ halo features are compiled in Table 2, which evaluated for the uppermost image segment. The scattering angle of the halo minimum $\left(\vartheta_{\text {halo, }}\right.$ in $)$ is smallest for the red channel and largest for the blue channel, which is responsible for the reddish inner edge and the slightly blueish outer edge of the $22^{\circ}$ halo visible in Fig. 4. It should be noted that in many cases the $22^{\circ}$ halo appears rather white apart from a slightly reddish inner edge 
(Minnaert, 1937; Vollmer, 2006). The differences between scattering angles for the three colors are smaller for $\vartheta_{\text {halo, max }}$, with a slightly larger value for the blue channel. The halo ratio amounts to about 1.15 averaged over all three channels and is largest for the green and smallest for the blue channel.

The angular position of the $22^{\circ}$ halo brightness peak $\left(\vartheta_{\text {halo, } \max }\right)$ can also be used to estimate the positioning accuracy of HaloCam relative to the sun. Figure 6 shows a histogram of $\vartheta_{\text {halo, max }}$ for 1289 randomly selected HaloCam pictures showing a $22^{\circ}$ halo in the uppermost image segment. This segment was chosen since it contains the most pronounced halos. For a faint halo the peak in the brightness distribution is rather flat, causing a larger uncertainty in finding the angular position of the peak. The mean value of $\vartheta_{\text {halo, max }}$ amounts to $21.9^{\circ}$ with a $2 \sigma$ standard deviation of $0.5^{\circ}$, which is a rough estimate of HaloCam's pointing accuracy. Since $\vartheta_{\text {halo, max }}$ and $\vartheta_{\text {halo, min }}$ are searched for within an angular interval, the pointing accuracy of $\pm 0.5^{\circ}$ is sufficient to detect the halo.

\section{Development of an automated halo detection algorithm}

The HaloCam long-term dataset from January 2014 until June 2016 was evaluated by applying a machine learning algorithm for the automated detection of halos. The algorithm was trained using features extracted from the HaloCam images. Some of these features (e.g., HR, $\vartheta_{\text {halo, max }}, \vartheta_{\text {halo, min }}$ ) were already described in the previous section. As a first implementation, the detection algorithm is presented here for the case of the $22^{\circ}$ halo, but it is possible to extend it to other halo types as well.

\subsection{Description of the classification algorithm}

The detection is performed by a classification algorithm which is trained to predict whether a HaloCam picture belongs to the class " $22^{\circ}$ halo" or "no $22^{\circ}$ halo". For such a binary classification a decision tree can be used to create a model which predicts the class of a data sample. Details on decision trees are explained in Appendix A. One major issue of decision trees is their tendency to overfit by growing arbitrarily complex trees depending on the complexity of the data. In this study we use the random forest classifier as described by Breiman (2001), which improves the issue of overfitting significantly by growing an ensemble of decision trees. A description of the random forest classifier used in this study is provided in Appendix B. In principle, other classification algorithms could be used, like artificial neural networks. The reasons why the random forest classifier was chosen are as follows. Apart from being robust against overfitting it does not require much preprocessing of the input data like scaling or normalizing. During the training of the individual trees the out-of-bag (OOB) samples (i.e., the sam- ples which were not in the training subsets) are used as test data, and classification error estimates (e.g., out-of-bag error) can be calculated simultaneously (Breiman, 2001). In contrast to an artificial neural network, the basic structure and the internal threshold tests of the decision trees are simple to understand and can be explained by boolean logic. Henceforward, the algorithm applied to the classification of $22^{\circ}$ halos will be called HaloForest.

The features used here for the classification are the $22^{\circ}$ halo ratio, the scattering angle position of the halo minimum and maximum, and the scattering angle confining the halo peak $\vartheta_{\text {halo, end }}$, which are shown in Fig. 7 together with the slope of the regression line in black (solid). The halo peak is confined by $\vartheta_{\text {halo, end }}$ (dash-dotted line), which represents the scattering angle with the same brightness level as $\vartheta_{\text {halo, min }}$ in the scattering angle interval $\left(\vartheta_{\text {halo, max }}, 35^{\circ}\right.$ ]. This feature is used to ensure that the brightness for angles larger than $\vartheta_{\text {halo, max }}$ is decreasing again. The slope of the regression line serves as an estimate for the brightness gradient around the sun. For clear-sky images this gradient is steeper than for overcast cases. As a measure of the separation of color in the halo, the scattering angle difference between the blue and red channel for the halo minimum $\left(\Delta \vartheta_{\text {halo, min }}\right)$ and maximum $\left(\Delta \vartheta_{\text {halo, max }}\right)$ are calculated, which are defined as

$$
\begin{aligned}
& \Delta \vartheta_{\text {halo, max }}=\vartheta_{\text {halo, max }} \text { blue }-\vartheta_{\text {halo, max, red }}, \\
& \Delta \vartheta_{\text {halo, min }}=\vartheta_{\text {halo, min, blue }}-\vartheta_{\text {halo, min, red }} .
\end{aligned}
$$

Furthermore, the standard deviation of the brightness averaged over the image segment is used as a proxy for the inhomogeneity of the scene. These eight features are calculated for each of the six image segments separately.

In order to get an impression of typical values of the training features for the two classes, Fig. 8a-c show twodimensional scatter plots of selected feature pairs for the upper image segment (no. 4). Features which belong to the class " $22^{\circ}$ halo" are displayed in blue, whereas the features of the class "no $22^{\circ}$ halo" are represented by gray scatter points. Figure $8 \mathrm{a}$ shows the distribution of the scattering angle of the halo maximum vs. minimum. The scattering angles of the halo maximum $\vartheta_{\text {halo, max }}$ are confined to a smaller interval for " $22^{\circ}$ halo" compared to "no $22^{\circ}$ halo". However, the two classes share many data points in this projection, so more features are needed to generate decision boundaries in a higher, here eight-dimensional, space. Figure $8 \mathrm{~b}$ depicts the scattering angle difference between the blue minus the red channel for the halo maximum $\left(\Delta \vartheta_{\text {halo, max }}\right)$ vs. minimum $\left(\Delta \vartheta_{\text {halo, max }}\right)$, which is positive for the " $22^{\circ}$ halo" class since the inner edge (smaller $\vartheta$ ) of the $22^{\circ}$ halo is slightly red. The $\mathrm{HR}$, which is shown in Fig. 8c, takes values between 1 and $\sim 1.3$ for " $22^{\circ}$ halos". Images with a low mean standard deviation of the image segment indicate rather homogeneous scenes which are present most of the time when a $22^{\circ}$ halo is visible. Figure $8 \mathrm{a}-\mathrm{c}$ visualize that the two classes, " $22^{\circ}$ halo" and "no $22^{\circ}$ halo", can not be separated easily since the val- 
(a)

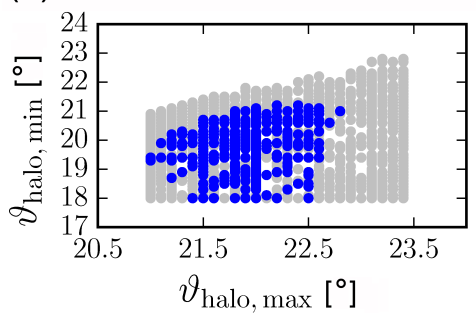

(d)

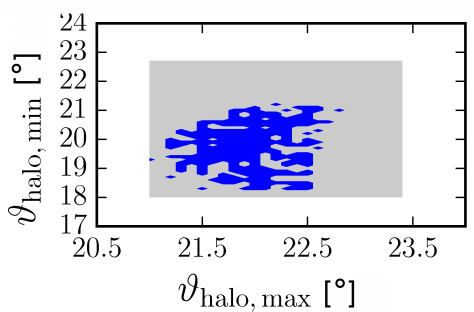

(b)

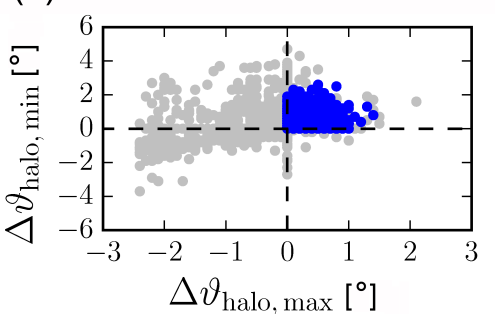

(e)

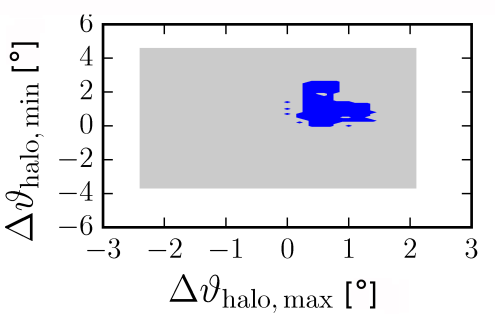

(c)

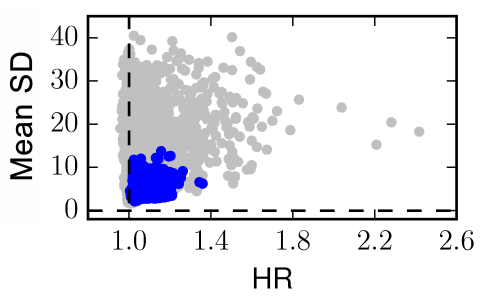

(f)

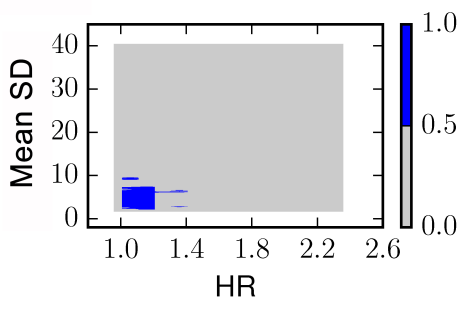

Figure 8. (a-c) Scatter plots of selected pairs of the eight features used for training HaloForest. Training samples with(out) $22^{\circ}$ halos are represented in blue (gray). (d-f) Decision boundaries of the random forest classifier for the respective feature pair. The predicted probability used for separating the classes " $22^{\circ}$ halo" $(p>0.5)$ and "no $22^{\circ}$ halo" $(p \leq 0.5)$ is displayed in blue and gray, respectively.

Table 3. Confusion matrix for HaloForest for the uppermost (no. 4) and lowermost (no. 1) image segments. The label "Predicted" refers to the class which was predicted by HaloForest, whereas "True" labels the visually identified class. The true positives (correctly classified " $22^{\circ}$ halo") are printed in bold font. False positives ("no $22^{\circ}$ halo" classified as " $22^{\circ}$ halo") and false negatives are listed on the other diagonal. The results are provided with a $2 \sigma$ standard deviation.

\begin{tabular}{llrr}
\hline & & \multicolumn{2}{c}{ Predicted } \\
\cline { 3 - 4 } & Segment 4: & $22^{\circ}$ halo & no $22^{\circ}$ halo \\
\hline \multirow{2}{*}{ True } & $22^{\circ}$ halo & $\mathbf{9 7 . 3} \pm \mathbf{1 . 9} \%$ & $0.4 \pm 0.3 \%$ \\
& no $22^{\circ}$ halo & $2.7 \pm 0.9 \%$ & $\mathbf{9 9 . 6} \pm \mathbf{0 . 2} \%$ \\
\hline \multirow{2}{*}{ True } & Segment 1: & $22^{\circ}$ halo & no $22^{\circ}$ halo \\
\hline & no $22^{\circ}$ halo & $\mathbf{8 8 . 5} \pm \mathbf{7 . 1} \%$ & $0.5 \pm 0.5 \%$ \\
& & & $\mathbf{9 9 . 5} \pm \mathbf{0 . 2} \%$ \\
\hline
\end{tabular}

ues of the features overlap. The lower panels of Fig. $8 \mathrm{~d}-\mathrm{f}$ display the regions which are detected as " $22^{\circ}$ halo" (blue) and "no $22^{\circ}$ halo" (gray) by the trained algorithm.

For each of the six image segments an individual classifier was trained using a dataset of visually classified HaloCam images which were chosen randomly from the dataset. The performance of the classifiers was tested using a random selection of $30 \%$ of the dataset which was excluded from training. This procedure was repeated 100 times to get statistically significant results for the performance of the classifier.

Table 3 shows the confusion matrix for the classifier of the segments directly above (no. 4) and below the sun (no. 1) which represent the two extreme cases of the performance of the six different classifiers: the upper part of the $22^{\circ}$ halo has a higher brightness contrast compared to the lower part which is often obstructed by the horizon. For the training of HaloForest 1289 samples with a $22^{\circ}$ halo and 5181 samples without $22^{\circ}$ halo were used for the uppermost segment (no. 4). The lowermost segment (no. 1) was trained with 296 and 3370 samples of the classes $22^{\circ}$ halo and no $22^{\circ}$ halo, respectively. The lines of the confusion matrix indicate the true class labels of the samples (" $22^{\circ}$ halo" and "no $22^{\circ}$ halo"), whereas the columns contain the predicted class labels. The number of true positive and negative (in bold) as well as false positive and negative classifications are evaluated and provided with a $2 \sigma$ standard deviation. The correct classification of " $22^{\circ}$ halo" is maximum for the uppermost image segment (no. 4) with about $98 \%$ and minimum for the lowermost segment with about $89 \%$. The correct classification of "no $22^{\circ}$ halo" is overall higher than $99 \%$, so the HaloForest algorithm seems to be able to separate the two classes well. The performance of the other four segments ranges between the results of the upper and lowermost segments.

\subsection{Application of the halo detection algorithm}

HaloForest is used to evaluate the dataset HaloCam collected in Munich between January 2014 and June 2016. To ensure a high classification accuracy, only the classifiers for the upper image segments (3,4 and 5) were used (cf. Table 3). A HaloCam image was assigned to the class " $22^{\circ}$ halo" if at least one of the image segments 3,4 , or 5 predicts a $22^{\circ}$ halo. Applying a probability threshold of $p>0.5,22^{\circ}$ halos were detected in $152 \mathrm{~h}$. Relative to the total observation time during daylight of $7345 \mathrm{~h}, 22^{\circ}$ halos occurred about $2.1 \%$ of 
Table 4. Confusion matrix as in Table 3 for 470 randomly selected HaloCam images between January 2014 and June 2016, evaluated for segments 3,4 and 5 . The true positives (correctly classified " $22^{\circ}$ halo") are printed in bold font.

\begin{tabular}{llrr}
\hline & \multicolumn{2}{c}{ Predicted } \\
\cline { 3 - 3 } & & $22^{\circ}$ halo & no $22^{\circ}$ halo \\
\hline \multirow{2}{*}{ True $\quad 22^{\circ}$ halo } & $\mathbf{8 8 . 8 \%}$ & $2.8 \%$ \\
& no $22^{\circ}$ halo & $11.2 \%$ & $\mathbf{9 7 . 2 \%}$ \\
\hline
\end{tabular}

the time. As an additional test, the classification accuracy of HaloForest was checked for 470 randomly chosen HaloCam images for the " $22^{\circ}$ halo" and "no $22^{\circ}$ halo" class within this long-term observation period in Munich. The confusion matrix for this test is provided in Table 4 for the image segments no. 3,4 and 5 together. More than $88 \%$ of the $22^{\circ}$ halos are classified correctly and less than $12 \%$ are classified incorrectly as $22^{\circ}$ halos.

Images were incorrectly classified as $22^{\circ}$ halo predominantly due to small bright clouds or contrails in a blue sky or structures in overcast conditions which happen to cause a peak in the averaged brightness distribution at a scattering angle of $22^{\circ}$.

Based on these results we investigated the fraction of cirrus clouds which produced a halo in Munich during this time period. The total frequency of occurrence of cirrus clouds was determined by independent data of colocated CHM15kx ceilometer observations (Wiegner and Geiß, 2012). To guarantee consistent observational conditions, only ceilometer measurements in the absence of lowlevel clouds were considered. Proprietary software of the ceilometer automatically provides up to three cloud base heights with a temporal resolution of $15 \mathrm{~s}$. The detection is based on the fact that in case of clouds backscatter signals are significantly larger than the background noise.

The sensitivity of the ceilometer is sufficient to even detect clouds near the tropopause during daytime. Since ceilometers, however, do not provide depolarization information, the discrimination between water and ice clouds was made by means of the cloud base temperature $T_{\text {base. Sassen and }}$ Campbell (2001) state that cirrus cloud base temperatures ranged between -30 and $-40{ }^{\circ} \mathrm{C}$ during the 10 -year observation period at the FARS observation site. As a temperature threshold is not an unambiguous criterion for the existence of ice clouds, we have calculated the frequency of occurrence for three different temperatures: $-20,-30$ and $-40^{\circ} \mathrm{C}$. If $T_{\text {base }}$ is lower than the given temperature threshold, the cloud is considered a "cirrus cloud". The temperature profiles were obtained from routine radiosonde ascents of the German Weather Service at Oberschleißheim (WMO station code 10868), which is located about $13 \mathrm{~km}$ north of the HaloCam site. During the time period from January 2014 until June 2016 a fraction of $5.6 \%$ cirrus clouds was detected

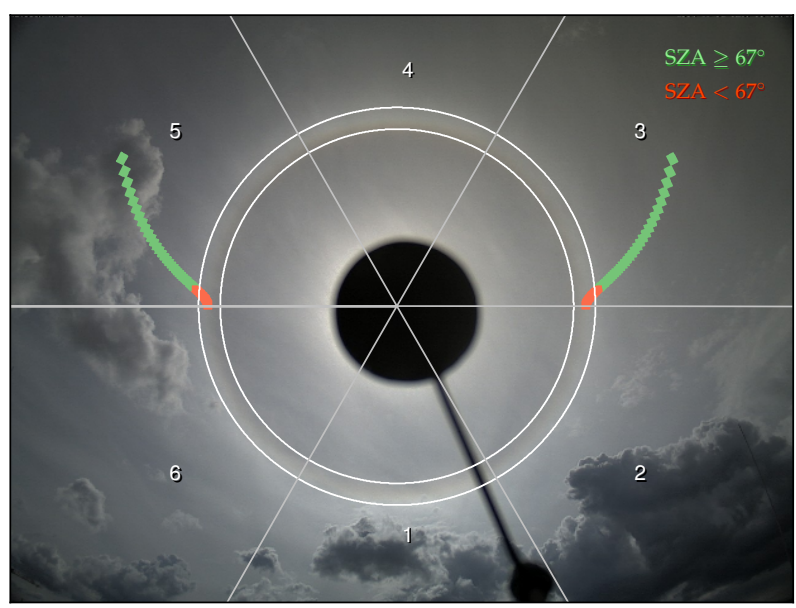

Figure 9. HaloCam image as in Fig. 4b. The red and green squares indicate the minimum scattering angle of the sundogs as a function of the solar zenith angle (SZA). The SZA ranges between 90 and $35^{\circ}$ with $1^{\circ}$ resolution. The mask used to search for the $22^{\circ}$ halo peak is displayed by the two white circles and covers scattering angles between 21.0 and $23.5^{\circ}$. Sundog positions located within this mask might be misclassified as $22^{\circ}$ halo and are marked as red. These positions correspond with SZAs between 90 and $67^{\circ}$. For smaller SZAs (higher solar elevations) the sundogs are located outside the mask and cannot be misclassified as $22^{\circ}$ halo by the algorithm.

for a cloud base temperature of $T_{\text {base }}<-20^{\circ} \mathrm{C}$. Towards lower cloud base temperatures the amount of detected cirrus clouds decreases to $3.5 \%$ for $T_{\text {base }}<-30^{\circ} \mathrm{C}$ and $1.9 \%$ for $T_{\text {base }}<-40^{\circ} \mathrm{C}$.

Due to the different pointing directions of the ceilometer (towards zenith) and HaloCam (towards sun), the instruments observe different regions of the sky. This is accounted for by prescreening the data for $1 \mathrm{~h}$ time intervals when the ceilometer detected a cirrus cloud. The prescreening is subject to data availability for both instruments. The subsequent analysis of cirrus fraction and halo frequency of occurrence is based on the full temporal resolution of 15 and $10 \mathrm{~s}$, respectively. Relative to the amount of detected cirrus clouds about $25 \%$ occurred together with a $22^{\circ}$ halo for the image segments 3 , 4 and 5. This fraction does not change much for the different cloud base temperatures $\left(26.4 \%\right.$ for $T_{\text {base }}<-20^{\circ} \mathrm{C}$ and $24.5 \%$ for $T_{\text {base }}<-40^{\circ} \mathrm{C}$ ) since the fraction of detected clouds decreases together with the detected halos for lower temperatures. According to the confusion matrix in Table 4, $88.8 \%$ of the detected " $22^{\circ}$ halos" are real halos, while $2.8 \%$ of the "no $22^{\circ}$ halos" are actually " $22^{\circ}$ halos". Correcting the result for the estimated false classifications, the fraction of "halo-producing" cirrus clouds amounts to about $25 \% \times 88.8 \%+75 \% \times 2.8 \% \approx 24 \%$. The comparison of the ceilometer and HaloCam data implies that about $25 \%$ of the cirrus clouds contain some fraction of smooth, hexagonal ice crystals. Sassen et al. (2003) observed a fraction of $37.3 \%$ 

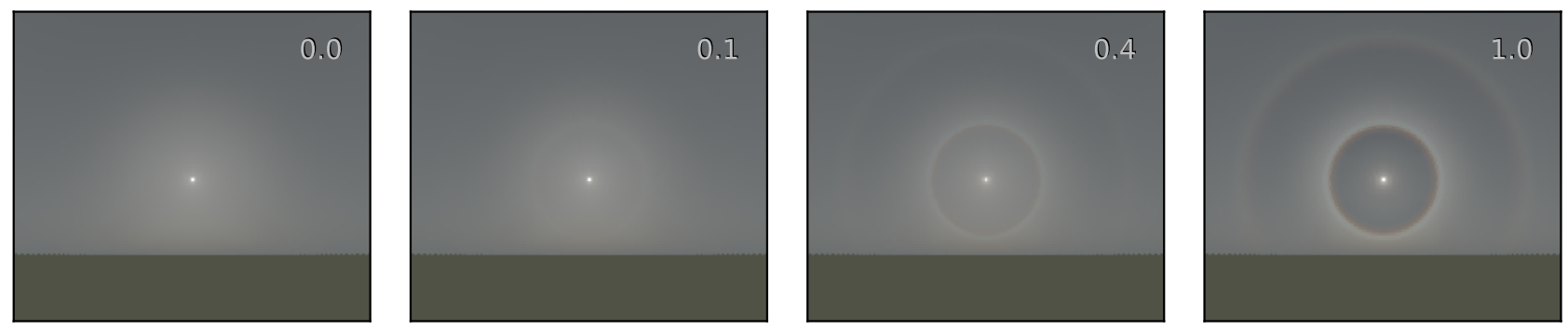

Figure 10. Sky radiance simulations with libRadtran (Mayer and Kylling, 2005) using the DISORT solver for a solar zenith angle of $60^{\circ}$, a viewing azimuth angle range of $0-160^{\circ}$ and for viewing zenith angles from $10-110^{\circ}$ (i.e., from the zenith to $20^{\circ}$ below the horizon). The simulations were performed for a spectral range of 380-780 $\mathrm{nm}(5 \mathrm{~nm}$ steps), weighted with the spectral sensitivity of the human eye. A homogeneous cirrus cloud layer with optical thickness of 1 was assumed. Solid column ice crystal optical properties of Yang et al. (2013) with an effective radius of $80 \mu \mathrm{m}$ were used. Aerosol scattering was not considered. The four panels show radiative transfer simulations with different fractions of smooth solid columns ranging from 0 to $100 \%$, as indicated by the labels. A background of severely roughened solid columns is assumed, with fractions changing from 100 to $0 \%$, accordingly.

cirrus clouds which produced a $22^{\circ}$ halo within $1 \mathrm{~h}$ time intervals. The results most likely differ because the observations originate from different locations which might be dominated by different mechanisms for cirrus formation. It has to be noted, however, that the evaluation method is very sensitive to the sampling strategy of the observations: the fraction of halo-producing cirrus clouds increases to more than $50 \%$ if the HaloCam observations are binned to $1 \mathrm{~h}$ intervals, which are counted as containing a halo regardless of their duration.

For comparison, the fraction of cirrus clouds producing a halo display was evaluated visually for the HaloCam observations during the ACCEPT campaign and amounts to about $27 \%$ including $22^{\circ}$ halos, sundogs and upper/lower tangent arcs (cf. Sect. 2.1). This value is also lower than the result provided by Sassen et al. (2003) who observed any of the three halo types in about $54 \%$ of the $1 \mathrm{~h}$ periods with cirrus.

The current version of HaloForest discriminates only between the two classes " $22^{\circ}$ halo" and "no $22^{\circ}$ halo". Thus, interference with other halo types as sundogs or upper/lower tangent arcs and circumscribed halos might occur at certain solar elevations. The position of sundogs relative to the sun depends on the solar zenith angle (SZA) and can be calculated analytically as described in Wegener (1925), Tricker (1970), Minnaert (1993), and Liou and Yang (2016). The sundogs are located at scattering angles close to the $22^{\circ}$ halo for large SZAs and occur at larger scattering angles for small SZAs, i.e., high solar elevations. Figure 9 shows the same HaloCam image with the azimuth segments as Fig. 4b. In addition, the minimum scattering angle of the sundogs are calculated as a function of the SZA and represented by the red and green squares. The SZAs range between 90 and $35^{\circ}$ with a resolution of $1^{\circ}$. The two white circles centered around the sun at scattering angles of 21.0 and $23.5^{\circ}$ indicate the mask which is used to find the scattering angle of the $22^{\circ}$ halo peak. For $\mathrm{SZA} \leq 67^{\circ}$ the sundog positions are located outside this mask and cannot be misclassified as $22^{\circ}$ halo (green squares). The red squares represent sundog positions which are located within this mask and might therefore be misclassified. This is the case for SZAs between 90 and $67^{\circ}$. To obtain an estimate of the fraction of sundogs which are misclassified as $22^{\circ}$ halo, 1000 randomly selected HaloCam images were counter-checked visually. This revealed that only six images showing sundogs without $22^{\circ}$ halo in the segments (3-5) were misclassified as $22^{\circ}$ halo, which is $<1 \%$. Upper tangent arcs could be detected by the uppermost image segment (no. 4) and might be misclassified as $22^{\circ}$ halo. For very small SZAs (high solar elevations) the tangent arcs merge to form the circumscribed halo which could be detected in the segments 3 and 5 as well. The same procedure was repeated for these halo types: 1000 randomly selected images were checked for the presence of tangent arcs and circumscribed halos without $22^{\circ}$ halo, yielding 28 images or $2.8 \%$. However, if only a fragment of a halo is visible in the uppermost segment, it is generally difficult to discriminate between an upper tangent arc or circumscribed halo and a $22^{\circ}$ halo.

The halo classification algorithm was presented for $22^{\circ}$ halos, but it is possible to include training data for other halo types as well. With the current version of HaloForest and the co-located ceilometer observations, the fraction of cirrus clouds producing a halo display was estimated to about $25 \%$ for Munich between January 2014 and September 2016. Extending HaloForest for the detection of other halo types, such as sundogs, the fraction of halo-producing cirrus clouds could easily exceed $25 \%$. In principle, HaloCam could also be equipped with a wide-angle lens to observe halo displays in a larger region of the sky, however, at the expense of spatial resolution.

\section{Sensitivity study of the visibility of the $22^{\circ}$ halo and interpretation of halo statistics}

In this section we discuss the factors that contribute to the visibility of halo displays using the example of the 
$22^{\circ}$ halo. This is important for a more detailed interpretation of the fraction of halo-producing cirrus clouds and ice crystal roughness.

The effect of varying cloud optical thickness on the visibility of halo displays has been already investigated by Kokhanovsky (2008), Gedzelman and Vollmer (2008), and Gedzelman (2008) using radiative transfer simulations. Kokhanovsky (2008) performed simulations of the brightness contrast of the $22^{\circ}$ halo as a function of the cirrus optical thickness using the radiative transfer model SCIATRAN neglecting molecular and aerosol scattering. The results show a linear decrease of the halo contrast with increasing optical thickness. Gedzelman (2008) and Gedzelman and Vollmer (2008) used the model HALOSKY for radiative transfer simulations of halos with varying cloud optical thickness. HALOSKY considers single scattering by air molecules, aerosol particles and cloud particles assuming homogeneous, plane-parallel atmospheric layers. Multiple scattering is calculated only within the cloud by a Monte Carlo subroutine. Gedzelman and Vollmer (2008) show results for radiance simulations of the $22^{\circ}$ halo in the principal plane below and above the sun. They found that the radiance at the bottom of the halo reaches a maximum value for smaller COT $(\approx 0.25)$ than the radiance at the top of the cloud $(\approx 0.63)$.

In this study, radiative transfer simulations were performed using the libRadtran radiative transfer package (Mayer and Kylling, 2005; Emde et al., 2016) and the DISORT (discrete ordinate technique) solver (Stamnes et al., 1988; Buras et al., 2011). LibRadtran allows for an accurate simulation of Rayleigh scattering, molecular absorption, aerosols, surface albedo, and water and ice clouds. DISORT is a one-dimensional solver regarding the atmosphere as a number of homogeneous, plane-parallel layers. Radiative transfer simulations of a cirrus cloud were performed assuming a homogeneous ice cloud layer with optical thickness 1 (at $550 \mathrm{~nm})$ at a height between 10 and $11 \mathrm{~km}$. Figure 10 shows simulations using different fractions of smooth solid columns $(0,10,40,100 \%)$ and assuming a background of severely roughened solid columns. All ice crystals have an effective radius of $80 \mu \mathrm{m}$. The optical properties were chosen from the database by Yang et al. (2013). The sun is located at a zenith angle of $60^{\circ}$. Sky radiance was calculated for an angular range between 0 and $160^{\circ}$ in the azimuth direction and $10-110^{\circ}$ (i.e., from $10^{\circ}$ off-zenith to $20^{\circ}$ below the horizon) in the zenith direction, which corresponds to the view of a wide-angle camera. The simulations were performed for a spectral range of 380-780 $\mathrm{nm}$ (5 nm steps), and the results were weighted with the spectral sensitivity of the human eye according to CIE (1986), as implemented in specrend (http://www.fourmilab.ch/documents/specrend/).

Aerosol scattering was not considered and a spectral surface albedo of grass was chosen (Feister and Grewe, 1995). For $0 \%$ (first panel of Fig. 10) all ice crystals are rough and thus no 22 or $46^{\circ}$ halo is visible. For a fraction of $10 \%$ smooth crystals, the $22^{\circ}$ halo starts to form, which is in agreement with the findings of van Diedenhoven (2014). The $46^{\circ}$ halo becomes visible for a fraction of $40 \%$ smooth crystals. For $100 \%$ smooth crystals both $22^{\circ}$ and $46^{\circ}$ halo reach a maximum brightness contrast for the respective cirrus optical thickness.

Figure 11 depicts the sensitivity of the halo brightness contrast, represented by the halo ratio as a function of the smooth ice crystal fraction (a), the aerosol optical thickness (AOT; b), the cirrus optical thickness (c), and the surface albedo (d) for a wavelength of $550 \mathrm{~nm}$. As in Fig. 10 a SZA of $60^{\circ}$ was chosen and the ice cloud was defined between $10-11 \mathrm{~km}$. The halo ratio was determined in the principal plane above the sun. The dashed lines indicate a halo ratio of 1 , which we defined as threshold for the visibility of halo displays. Figure 11a shows clearly that for a smooth crystal fraction of $>10 \%$ the halo ratio exceeds 1 and the $22^{\circ}$ halo is visible. An increasing aerosol optical thickness causes a decrease of the HR, which is displayed in Fig. 11b. For a typical value of $\mathrm{AOT}=0.2$, the HR is reduced by $\sim 10 \%$ compared to an aerosol free atmosphere. Figure 11c illustrates how the HR is determined by the optical thickness of the cirrus cloud (COT) itself. We observe a maximum value for COT $\sim 1$. For a very thin cirrus, Rayleigh and aerosol scattering become dominant, resulting in a small HR. The HR approaches its maximum value only when COT is larger than the optical thickness of the background (here Rayleigh and aerosol).

For large COT, multiple scattering reduces the contrast of the halo feature and the HR decreases, similar to the findings of Kokhanovsky (2008). However, as Gedzelman and Vollmer (2008) point out, the halo peak might still be visible up to an optical thickness of $\sim 5$ due to the pronounced maximum in the scattering phase function.

A higher surface albedo causes longer photon paths through the atmosphere and thus a higher chance of multiple scattering (Fig. 11d). Reflected photons therefore cause a higher "background" brightness. It is evident that a brighter background causes a weaker brightness contrast of the halo display. In general, the effect of the surface albedo on the HR is small compared to the effect of AOT or COT. Halo displays are a geometric optics phenomenon, which means that they emerge only when the particle size is much larger than the wavelength (Fraser, 1979; Mishchenko and Macke, 1999; Garrett et al., 2007; Flatau and Draine, 2014), which also depends on the aspect ratio of the crystals (Um and McFarquhar, 2015). The solar zenith angle affects the halo brightness contrast indirectly by increasing the photon path length through the atmosphere for large SZAs and thus increasing the amount of multiple scattering (not shown). This effect is the same for different viewing zenith angles, which explains why the $22^{\circ}$ halo is always brightest at the top (directly above the sun) and faintest below the sun.

With this knowledge we can now discuss further implications of the fraction of halo-producing cirrus clouds. HaloCam observations showed that $\sim 25 \%$ of the cirrus clouds, 
(a)

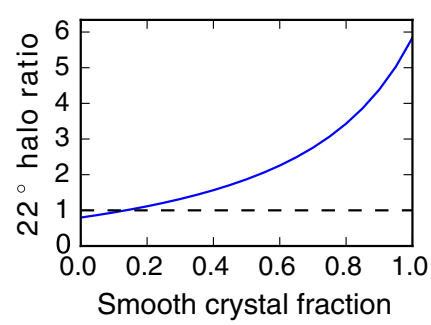

(b)

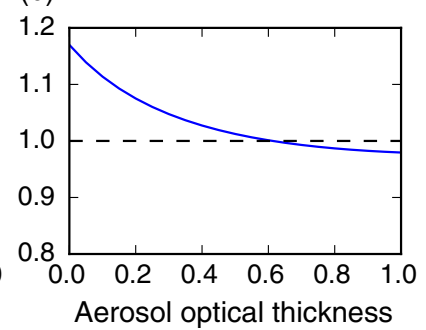

(c)

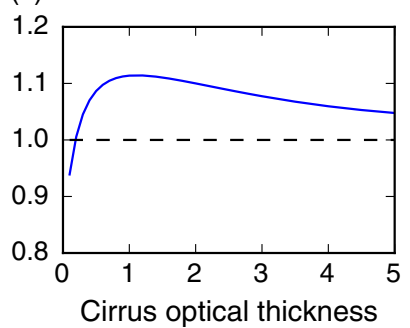

(d)

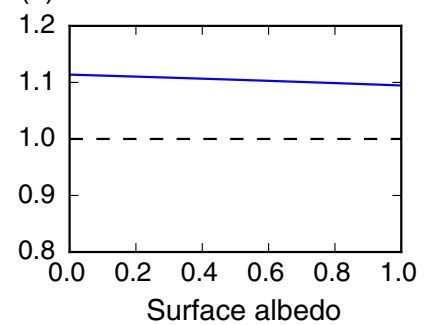

Figure 11. Sensitivity studies of the $22^{\circ}$ halo ratio at $550 \mathrm{~nm}$ (as defined in Eq. 1) as a function of smooth crystal fraction, aerosol optical thickness (AOT), cirrus optical thickness (COT) and surface albedo (from left to right). The radiative transfer simulations were performed with libRadtran assuming an ice cloud between 10 and $11 \mathrm{~km}$ using ice crystal optical properties as in Fig. 10 for a solar zenith angle of $60^{\circ}$. The dashed line indicates $\mathrm{HR}=1$, which marks the threshold for the visibility of a halo display. The default parameters, i.e., if not varied, are $20 \%$ smooth solid columns, AOT $=0.2, \mathrm{COT}=1.0$ and albedo $=0.0$.

which were visible from the ground, produced a $22^{\circ}$ halo. It can be argued that these cirrus clouds contained a certain amount of smooth, hexagonal ice crystals. By analyzing ice crystal single scattering properties, van Diedenhoven (2014) showed that a minimum fraction of $10 \%$ smooth hexagonal ice crystal columns is sufficient to produce a $22^{\circ}$ halo. With about $40 \%$, the minimum fraction of smooth crystals is much larger in the case of ice crystal plates for a visible halo. Thus, if the exact ice crystal habits of the cirrus cloud are unknown, which is typically the case, the minimum amount of smooth ice crystals probably lies in a range of 10 to $40 \%$. This implies that even for a large fraction of irregular or small ice crystals a halo might still be visible. A larger fraction of smooth ice crystals, however, could well be possible for halos with larger HR, i.e., increased brightness contrast. Multiple scattering of the cirrus cloud or atmosphere was not considered by van Diedenhoven (2014). This study revealed that during the $\sim 2.5$ years of HaloCam observations in Munich about $75 \%$ of the cirrus clouds did not produce a $22^{\circ}$ halo. For favorable atmospheric conditions, i.e., COT $\sim 1$ and negligible aerosol scattering, the maximum fraction of rough ice crystals ranges between 60 and $90 \%$. Thus, it is possible that the majority of cirrus clouds during the observation period in Munich contain a large fraction of rough ice crystals. This would support the hypothesis of Knap et al. (2005), Baran and Labonnote (2006), and Baran et al. (2015), who found that on average rough ice crystals better reproduce remote sensing radiance measurements than assuming crystals with smooth surface. However, if multiple scattering by cirrus clouds or aerosol is accounted for, the minimum fraction of smooth crystals could be much larger in the case of haloproducing cirrus clouds. The actual fraction of smooth ice crystals for cirrus clouds with visible halo display must be analyzed in detail and will be addressed in future work. This requires HaloCam observations to be complemented by radiative transfer simulations and additional measurements of aerosol and cirrus optical thickness. These additional measurements can be provided by radar, lidar and sun photome- ter measurements available at the observation site at MIM, LMU, in Munich. Surface albedo measurements can be obtained from satellite data products.

\section{Summary and conclusions}

In this paper we present HaloCam, a novel sun-tracking camera system for the automated observation of halo displays. The camera has a field of view of $90^{\circ}$ in the horizontal and $67^{\circ}$ in the vertical direction and a resolution of $1280 \times 960$ quadratic pixels which yields an angular resolution of $0.07^{\circ}$. The camera system records images in RGB color space and JPEG compression every $10 \mathrm{~s}$. It automatically tracks the sun so that the halo displays stay centered relative to the camera. HaloCam observations can contribute to a better understanding of ice crystal shape, surface roughness and orientation by long-term observations of halo displays. Different halo displays are caused by different ice crystal shapes and orientations. The most frequent halo displays are formed by either randomly oriented or oriented plates and columns and therefore contain the most important information about ice crystal properties. Therefore, the camera setup was optimized for observing $22^{\circ}$ halos, sundogs, and upper/lower tangent arcs or circumscribed halos with high spatial and temporal resolution without loosing relevant information.

An initial visual evaluation of the frequency of halo displays reveals that for the 6-week ACCEPT campaign sundogs were observed more often than $22^{\circ}$ halos. Together with the observations of upper tangent arcs, this implies that about $73 \%$ of the observed halo displays were caused by oriented ice crystals. This result differs from the findings of other studies, like Sassen et al. (2003), which observed that $22^{\circ}$ halos are more frequent than sundogs and upper tangent arcs based on a dataset of about 10 years. A visual evaluation of the presence of cirrus clouds during the campaign showed that about $27 \%$ produced a $22^{\circ}$ halo, sundogs, or upper/lower tangent arcs. Sassen et al. (2003) found that in about $54 \%$ of the $1 \mathrm{~h}$ cirrus periods at least one of the three halo types was 
visible. It should be highlighted that the evaluation method is very sensitive to the sampling method and the temporal resolution of the observations.

For evaluating the long-term HaloCam observations in Munich, an automated halo detection algorithm, called HaloForest, was developed. HaloForest is presented here for the detection of $22^{\circ}$ halos, but it can be extended for the detection of other halo types such as sundogs and upper/lower tangent arcs. The algorithm is based on a random forest classifier and was trained and tested against visually evaluated observations. With more than $88 \%$ of the test samples correctly classified as " $22^{\circ}$ halos" and more than $97 \%$ correctly classified as "no $22^{\circ}$ halo", HaloForest is able to separate the two classes well. Applied to the more than 2.5 years of data, HaloForest detected $22^{\circ}$ halos about $2 \%$ of the total observation time during daylight.

A first estimate of ice crystal roughness was performed by evaluating the frequency of cirrus clouds that were accompanied by halo displays. For the long-term halo observations in Munich, co-located ceilometer measurements were used to evaluate the fraction of cirrus clouds. About $25 \%$ of the detected cirrus clouds in Munich occurred together with a $22^{\circ}$ halo. Extending HaloForest for more halo types (e.g., sundogs) would increase the fraction of halo-producing cirrus clouds above $25 \%$.

These results imply that the majority of cirrus clouds which did not produce a visible halo, very likely, contained primarily rough ice crystals and $25 \%$ (or $27 \%$ for ACCEPT) of the clouds contained at least a certain fraction of smooth, hexagonal ice crystals. Based on the study by van Diedenhoven (2014) a minimum fraction of smooth crystals of $10 \%$ in case of columns or $40 \%$ in case of plates can be estimated for the halo-producing cirrus clouds if multiple scattering and scattering by aerosol is neglected. These assumptions allow determining a minimum fraction of smooth crystals in haloproducing cirrus clouds. If multiple scattering by cloud and aerosol is accounted for, the required fraction of smooth ice crystals could be significantly larger than $40 \%$. To further constrain the fraction of rough ice crystals, more detailed quantitative studies are needed, which will be addressed in future work. This analysis requires radiative transfer simulations and additional constraints which can be provided by radar, lidar and sun photometer measurements available at the observation site at LMU in Munich.

This study highlights the potential and feasibility of a completely automated method to collect and evaluate halo observations. These long-term observations allow estimating the average fraction of rough ice crystals in cirrus clouds. Quantitative evaluation of halo radiance distributions can contribute to systematically investigate ice crystal surface roughness, shape and orientation in cirrus clouds. Implemented on different sites, HaloCam in combination with the HaloForest detection algorithm can provide a consistent dataset for climatological studies.
Data availability. The radiosonde data are available via the website of the University of Wyoming, College of Engineering, Department of Atmospheric Science, at http://weather.uwyo.edu/upperair/ sounding.html. Due to the large file size, the HaloCam images and the ceilometer data from the measurement site at the Meteorological Institute (LMU) in Munich from January 2014 until June 2016 will be provided upon request. A sample HaloCam image is provided in the Supplement, which is the underlying source of the data of Table 2 and Figs. 4, 5, 7 and 9.

The Supplement related to this article is available online at https://doi.org/10.5194/amt-10-2499-2017-supplement. 


\section{Appendix A: Decision trees}

The subsequent sections provide more details on decision trees and the random forest classifier presented in Sect. 3.

The following description is based on Alpaydin (2010) and Raschka (2015). Decision trees start with a root node followed by internal decision nodes, branches and terminal nodes, called leaves. A typical example of a single decision tree, as used for HaloForest, is shown in Fig. A1. For a better visualization, the tree is grown using only three of the eight features and is pruned to a depth of three layers. The explanation provided here focuses on the structure of tree rather than the exact numbers of the threshold tests which differ from the ones used by HaloForest. The halo ratio (HR), the mean standard deviation and $\Delta \vartheta_{\text {halo, min }}$ are used as features in this case, which are displayed in the first line of each node box with the respective threshold test. At each decision node a threshold test is applied to one element of the $n$-dimensional feature vector (here, $n=3$ ) which best splits the set of samples. The metric to determine the best split in this study is the Gini impurity index, which is defined by Raschka (2015) as

$I_{\mathrm{G}}(t)=1-\sum_{i=1}^{c} p(i \mid t)^{2}$,

with $c$ being the number of classes and $p(i \mid t)$ the fraction of samples which belongs to class $i$ at node $t$. The Gini index takes a minimum value for the maximum information gain (all the samples at node $t$ belong to one class), and the index is maximum for a uniform distribution. The discrete result (here, true or false) of the threshold test decides which of the following branches is chosen. The node boxes are connected by arrows representing the branches of the tree. They are colored depending on the dominating class in the samples, which is noted at the bottom of each box: red for " $22^{\circ}$ halo" and blue for "no $22^{\circ}$ halo". The more transparent the color the higher the impurity of the classes and the larger the Gini impurity index. This splitting process is repeated recursively at each child node until a leaf node is reached. A leaf node is hit when all the samples in the subset belong to the same class or when splitting does not add more information. By repeating this recursive decision process, the $n$-dimensional feature space is subdivided into the predefined classes on a path following from the root down. Figure 8 shows examples of the resulting decision boundaries as two-dimensional projections for a selection of feature pairs. The decision tree is trained using a set of labeled training samples. During training the tree grows by adding branches and leaves depending on the complexity of the data, which can lead to overfitting. By growing an ensemble of decision trees, this issue can be improved, which is the idea of random forest classifiers.

\section{Appendix B: Random forest classifier implementation}

In this study we use the random forest classifier, which is described by Breiman (2001) and implemented in the python module scikit-learn (Pedregosa et al., 2011, version 0.18.1). The trees are trained by applying the bootstrap aggregation (bagging) method (Breiman, 1996), i.e., by using a subset of the training samples which is chosen randomly with replacement and has the same size as the original input samples. This implementation predicts the class of a sample by averaging the probabilistic prediction of all individual decision trees instead of using the majority vote among the trees. The function call allows the definition of a number of parameters: the number of trees is set to 100 and a maximum number of three features $\left(\log _{2}(n)\right.$ with $n$ features) is considered for searching the best split. These parameters are chosen to minimize the out-of-bag (OOB) error, as shown in Fig. B1.

For an increasing number of estimators (trees), the OOB error stabilizes for around 100 trees and is, in general, smaller for a confined number of features considered at each split. 


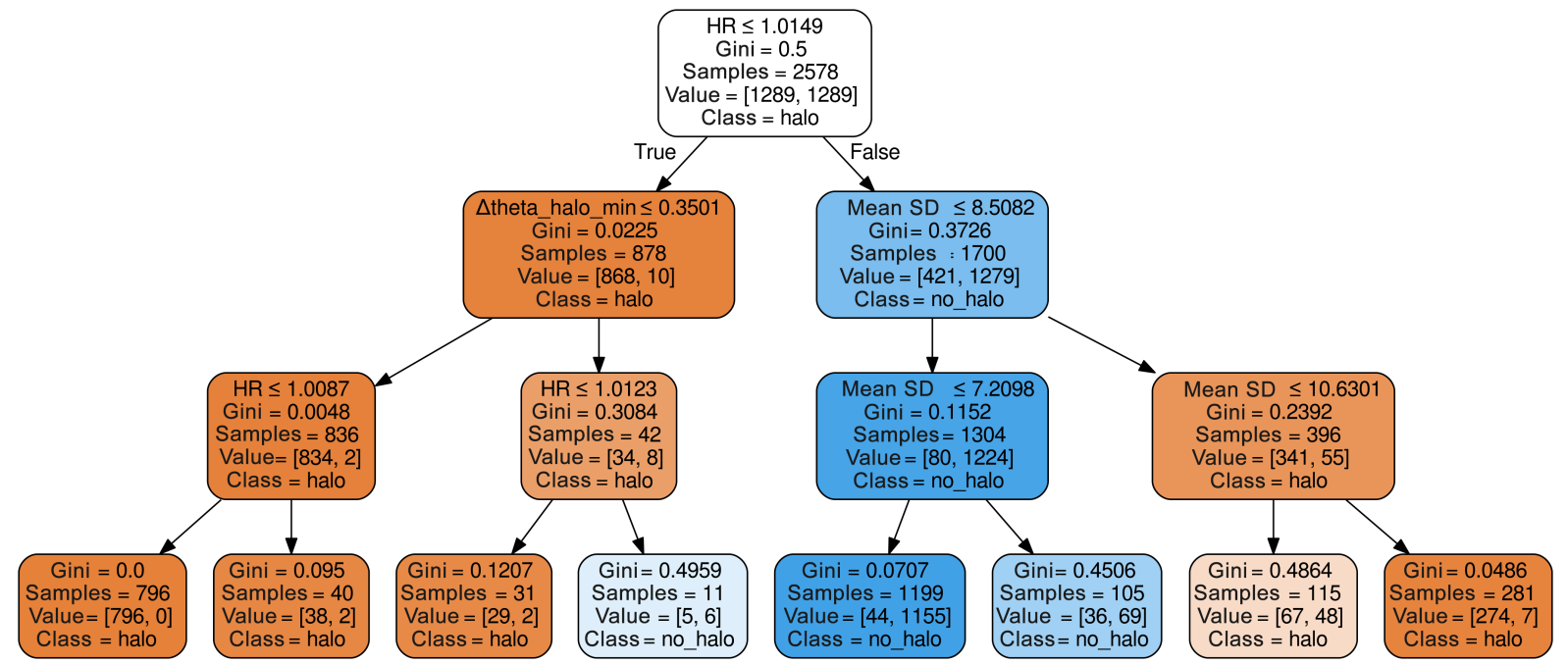

Figure A1. Example for a decision tree for a selection of three HaloCam image features confined to a maximum depth of three layers. The two classes, "halo" and "no halo", are depicted by red and blue color. The transparency of the color represents the impurity of the class.

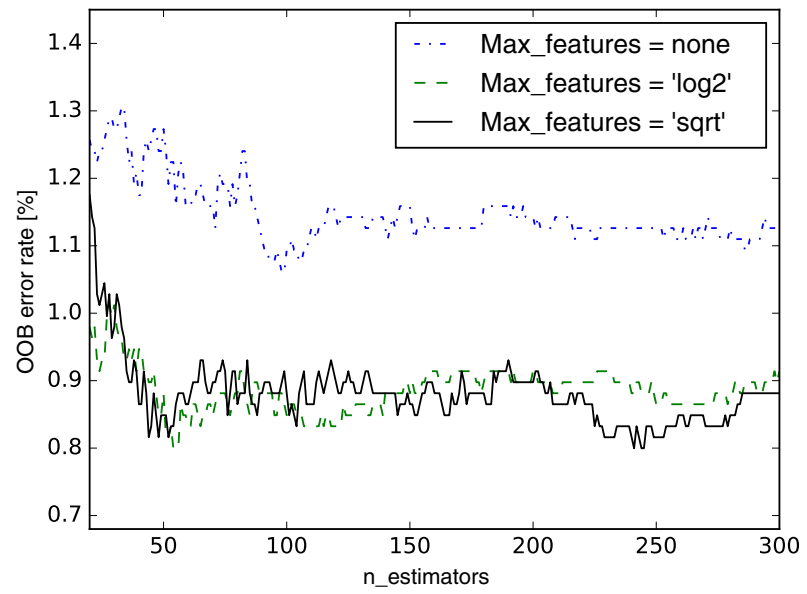

Figure B1. Out-of-bag error for different values of n_estimators (number of trees) for three different realizations of the random forest classifier by changing the number of features considered at each split. 
Competing interests. The authors declare that they have no conflict of interest.

Acknowledgements. The radiosonde data were downloaded from http://weather.uwyo.edu/upperair/sounding.html from the University of Wyoming, College of Engineering, Department of Atmospheric Science. The halo observations during the ACCEPT campaign research received funding from the European Union Seventh Framework Program (FP7/2007-2013) under grant agreement no 262254. We thank Markus Garhammer (LMU, Munich) and Marc Allaart (KNMI, the Netherlands) for their support during the campaign.

Edited by: Murray Hamilton

Reviewed by: Bastiaan van Diedenhoven

and two anonymous referees

\section{References}

Alpaydin, E.: Introduction to Machine Learning, Adaptive Computation and Machine Learning, 2nd edn., MIT Press, Cambridge, 2010.

Baran, A. J. and Labonnote, L. C.: On the reflection and polarisation properties of ice cloud, J. Quant. Spectrosc. Ra., 100, 41-54, https://doi.org/10.1016/j.jqsrt.2005.11.062, 2006.

Baran, A. J., Furtado, K., Labonnote, L.-C., Havemann, S., Thelen, J.-C., and Marenco, F.: On the relationship between the scattering phase function of cirrus and the atmospheric state, Atmos. Chem. Phys., 15, 1105-1127, https://doi.org/10.5194/acp15-1105-2015, 2015.

Bradski, D. G. R. and Kaehler, A.: Learning Opencv, 1st edn., O'Reilly Media, Inc., Sebastopol, 2008.

Breiman, L.: Bagging Predictors, Mach. Learn., 24, 123-140, https://doi.org/10.1023/A:1018054314350, 1996.

Breiman, L.: Random forests, Mach. Learn., 45, 5-32, https://doi.org/10.1023/A:1010933404324, 2001.

Buras, R., Dowling, T., and Emde, C.: New secondary-scattering correction in DISORT with increased efficiency for forward scattering, J. Quant. Spectrosc. Ra., 112, 2028-2034, 2011.

CIE: Standard on Colorimetric Observers, Commission Internationale de l'Eclairage CIE, 1986.

Emde, C., Buras-Schnell, R., Kylling, A., Mayer, B., Gasteiger, J., Hamann, U., Kylling, J., Richter, B., Pause, C., Dowling, T., and Bugliaro, L.: The libRadtran software package for radiative transfer calculations (version 2.0.1), Geosci. Model Dev., 9, 1647-1672, https://doi.org/10.5194/gmd-9-1647-2016, 2016.

Feister, U. and Grewe, R.: Spectral albedo measurements in the UV and visible region over different types of surfaces, Photochem. Photobiol., 62, 736-744, https://doi.org/10.1111/j.17511097.1995.tb08723.x, 1995.

Flatau, P. J. and Draine, B. T.: Light scattering by hexagonal columns in the discrete dipole approximation, Opt. Express, 22, 21834-21846, https://doi.org/10.1364/OE.22.021834, 2014.

Fraser, A. B.: What size of ice crystals causes the halos?, J. Opt. Soc. Am., 69, 1112-1118, https://doi.org/10.1364/JOSA.69.001112, 1979.
Garrett, T. J., Kimball, M. B., Mace, G. G., and Baumgardner, D. G.: Observing cirrus halos to constrain in-situ measurements of ice crystal size, Atmos. Chem. Phys. Discuss., 7, 1295-1325, https://doi.org/10.5194/acpd-7-1295-2007, 2007.

Gayet, J.-F., Mioche, G., Shcherbakov, V., Gourbeyre, C., Busen, R., and Minikin, A.: Optical properties of pristine ice crystals in mid-latitude cirrus clouds: a case study during CIRCLE-2 experiment, Atmos. Chem. Phys., 11, 2537-2544, https://doi.org/10.5194/acp-11-2537-2011, 2011.

Gedzelman, S. D.: Simulating halos and coronas in their atmospheric environment, Appl. Optics, 47, H157-H166, https://doi.org/10.1364/AO.47.00H157, 2008.

Gedzelman, S. D. and Vollmer, M.: Atmospheric optical phenomena and radiative transfer, B. Am. Meteorol. Soc., 89, 471-485, https://doi.org/10.1175/BAMS-89-4-471, 2008.

Görsdorf, U., Lehmann, V., Bauer-Pfundstein, M., Peters, G., Vavriv, D., Vinogradov, V., and Volkov, V.: A 35-GHz polarimetric doppler radar for long-term observations of cloud parameters - description of system and data processing, J. Atmos. Ocean. Tech., 32, 675-690, https://doi.org/10.1175/JTECH-D14-00066.1, 2015.

Greenler, R.: Rainbows, halos and glories, Cambridge University Press, Cambridge, 1980.

Heikkila, J. and Silven, O.: A four-step camera calibration procedure with implicit image correction, in: Proceedings of IEEE Computer Society Conference on Computer Vision and Pattern Recognition, 17-19 June 1997, San Juan, Puerto Rico, USA, 1106-1112, https://doi.org/10.1109/CVPR.1997.609468, 1997.

Holben, B., Eck, T., Slutsker, I., Tanré, D., Buis, J., Setzer, A., Vermote, E., Reagan, J., Kaufman, Y., Nakajima, T., Lavenu, F., Jankowiak, I., and Smirnov, A.: AERONET - a federated instrument network and data archive for aerosol characterization, Remote Sens. Environ., 66, 1-16, https://doi.org/10.1016/S00344257(98)00031-5, 1998.

Itseez: Open Source Computer Vision Library, available at: https: //github.com/itseez/opencv (10 July 2017), 2015.

Knap, W. H., Labonnote, L. C., Brogniez, G., and Stammes, P.: Modeling total and polarized reflectances of ice clouds: evaluation by means of POLDER and ATSR-2 measurements, Appl. Optics, 44, 4060-4073, https://doi.org/10.1364/AO.44.004060, 2005.

Kokhanovsky, A.: The contrast and brightness of halos in crystalline clouds, Atmos. Res., 89, 110-112, https://doi.org/10.1016/j.atmosres.2007.12.006, 2008.

Liou, K. and Yang, P.: Light Scattering by Ice Crystals: Fundamentals and Applications, Cambridge University Press, Cambridge, 2016.

Lynch, D. K. and Schwartz, P.: Intensity profile of the $22^{\circ}$ halo, J. Opt. Soc. Am. A, 2, 584-589, https://doi.org/10.1364/JOSAA.2.000584, 1985.

Mayer, B. and Kylling, A.: Technical note: The libRadtran software package for radiative transfer calculations - description and examples of use, Atmos. Chem. Phys., 5, 1855-1877, https://doi.org/10.5194/acp-5-1855-2005, 2005.

Minnaert, M.: De natuurkunde van 't vrije veld. Deel I. Licht en kleur in het landschap, W. J. Thieme, Zutphen, 1937.

Minnaert, M.: Rainbows, Halos, and Coronas, Springer New York, New York, NY, 185-258, https://doi.org/10.1007/978-1-46122722-9_10, 1993. 
Mishchenko, M. and Macke, A.: How big should hexagonal ice crystals be to produce halos?, Appl. Optics, 38, 1626-1629, https://doi.org/10.1364/AO.38.001626, 1999.

Myagkov, A., Seifert, P., Wandinger, U., Bühl, J., and Engelmann, R.: Relationship between temperature and apparent shape of pristine ice crystals derived from polarimetric cloud radar observations during the ACCEPT campaign, Atmos. Meas. Tech., 9, 3739-3754, https://doi.org/10.5194/amt-9-3739-2016, 2016.

Pedregosa, F., Varoquaux, G., Gramfort, A., Michel, V., Thirion, B., Grisel, O., Blondel, M., Prettenhofer, P., Weiss, R., Dubourg, V., Vanderplas, J., Passos, A., Cournapeau, D., Brucher, M., Perrot, M., and Duchesnay, E.: Scikit-learn: machine learning in Python, J. Mach. Learn. Res., 12, 2825-2830, 2011.

Pekkola, M.: Finnish Halo Observing Network: search for rare halo phenomena, Appl. Optics, 30, 3542-3544, https://doi.org/10.1364/AO.30.003542, 1991.

Pernter, J. M. and Exner, F.: Meteorologische Optik, W. Braumüller, Wien, 1910.

Raschka, S.: Python Machine Learning, Community experience distilled, Packt Publishing, Birmingham, 2015.

Sassen, K. and Campbell, J. R.: A midlatitude cirrus cloud climatology from the facility for atmospheric remote sensing. Part I: Macrophysical and synoptic properties, J. Atmos. Sci., 58, 481-496, https://doi.org/10.1175/15200469(2001)058<0481:AMCCCF>2.0.CO;2, 2001.

Sassen, K., Zhu, J., and Benson, S.: Midlatitude cirrus cloud climatology from the facility for atmospheric remote sensing. IV. Optical displays, Appl. Optics, 42, 332-341, https://doi.org/10.1364/AO.42.000332, 2003.

Seefeldner, M., Oppenrieder, A., Rabus, D., Reuder, J., Schreier, M., Hoeppe, P., and Koepke, P.: A two-axis tracking system with datalogger, J. Atmos. Ocean. Tech., 21, 975-979, https://doi.org/10.1175/15200426(2004)021<0975:ATTSWD>2.0.CO;2, 2004.

Shcherbakov, V.: Why the $46^{\circ}$ halo is seen far less often than the $22^{\circ}$ halo?, J. Quant. Spectrosc. Ra., 124, 37-44, https://doi.org/10.1016/j.jqsrt.2013.03.002, 2013.

Stamnes, K., Tsay, S., Wiscombe, W., and Jayaweera, K.: A numerically stable algorithm for discrete-ordinate-method radiative transfer in multiple scattering and emitting layered media, Appl. Optics, 27, 2502-2509, https://doi.org/10.1364/AO.27.002502, 1988.

Tape, W.: Atmospheric halos, Antarctic Research Series, American Geophysical Union, Washington DC, 1994.

Tape, W. and Moilanen, J.: Atmospheric Halos and the Search for Angle X, American Geophysical Union, Washington DC, 2006.

Toledano, C., Wiegner, M., Garhammer, M., Seefeldner, M., Gasteiger, J., Müller, D., and Koepke, P.: Spectral aerosol optical depth characterization of desert dust during SAMUM 2006, Tellus B, 61, 216-228, https://doi.org/10.1111/j.16000889.2008.00382.x, 2009.

Toledano, C., Wiegner, M., Groß, S., Freudenthaler, V., Gasteiger, J., Müller, D., Müller, T., Schladitz, a., Weinzierl, B., Torres, B., and O'Neill, N. T.: Optical properties of aerosol mixtures derived from sun-sky radiometry during SAMUM2, Tellus B, 63, 635-648, https://doi.org/10.1111/j.16000889.2011.00573.x, 2011.

Tricker, R. A. R.: Introduction to Meteorological Optics, Elsevier, New York, 1970.
Um, J. and McFarquhar, G. M.: Formation of atmospheric halos and applicability of geometric optics for calculating singlescattering properties of hexagonal ice crystals: Impacts of aspect ratio and ice crystal size, J. Quant. Spectrosc. Ra., 165, 134-152, https://doi.org/10.1016/j.jqsrt.2015.07.001, 2015.

van Diedenhoven, B.: The prevalence of the $22^{\circ}$ halo in cirrus clouds, J. Quant. Spectrosc. Ra., 146, 475-479, https://doi.org/10.1016/j.jqsrt.2014.01.012, 2014.

Venn, J.: On the employment of geometrical diagrams for the sensible representations of logical propositions, P. Camb. Philos. Soc., 4, 47-59, 1880.

Verschure, P.-P. H.: Thirty years of observing and documenting sky optical phenomena, Appl. Optics, 37, 1585-1588, https://doi.org/10.1364/AO.37.001585, 1998.

Voigt, C., Schumann, U., Minikin, A., Abdelmonem, A., Afchine, A., Borrmann, S., Boettcher, M., Buchholz, B., Bugliaro, L., Costa, A., Curtius, J., Dollner, M., Dörnbrack, A., Dreiling, V., Ebert, V., Ehrlich, A., Fix, A., Forster, L., Frank, F., Fütterer, D., Giez, A., Graf, K., Grooß, J.-U., Groß, S., Heimerl, K., Heinold, B., Hüneke, T., Järvinen, E., Jurkat, T., Kaufmann, S., Kenntner, M., Klingebiel, M., Klimach, T., Kohl, R., Krämer, M., Krisna, T. C., Luebke, A., Mayer, B., Mertes, S., Molleker, S., Petzold, A., Pfeilsticker, K., Port, M., Rapp, M., Reutter, P., Rolf, C., Rose, D., Sauer, D., Schäfler, A., Schlage, R., Schnaiter, M., Schneider, J., Spelten, N., Spichtinger, P., Stock, P., Walser, A., Weigel, R., Weinzierl, B., Wendisch, M., Werner, F., Wernli, H., Wirth, M., Zahn, A., Ziereis, H., and Zöger, M.: ML-CIRRUS: the airborne experiment on natural cirrus and contrail cirrus with the high-altitude long-range research aircraft HALO, B. Am. Meteorol. Soc., 98, 271-288, https://doi.org/10.1175/BAMS-D-1500213.1, 2017.

Vollmer, M.: Lichtspiele in der Luft, 1st edn., Spektrum Akademischer Verlag, München, 2006.

Wegener, A.: Theorie der Haupthalos, Aus dem Archiv der Deutschen Seewarte und des Marineobservatoriums, Hamburg, 43, 1925.

Wendisch, M., Yang, P., and Pilewskie, P.: Effects of ice crystal habit on thermal infrared radiative properties and forcing of cirrus, J. Geophys. Res., 112, D08201, https://doi.org/10.1029/2006JD007899, 2007.

Wiegner, M. and Geiß, A.: Aerosol profiling with the Jenoptik ceilometer CHM15kx, Atmos. Meas. Tech., 5, 1953-1964, https://doi.org/10.5194/amt-5-1953-2012, 2012.

Wiegner, M., Madonna, F., Binietoglou, I., Forkel, R., Gasteiger, J., Geiß, A., Pappalardo, G., Schäfer, K., and Thomas, W.: What is the benefit of ceilometers for aerosol remote sensing? An answer from EARLINET, Atmos. Meas. Tech., 7, 1979-1997, https://doi.org/10.5194/amt-7-1979-2014, 2014.

Wylie, D., Menzel, W., Woolf, H., and Strabala, K.: Four years of global cirrus cloud statistics using HIRS, J. Climate, 7, 19721986, 1994.

Yang, P., Bi, L., Baum, B. A., Liou, K.-N., Kattawar, G. W., Mishchenko, M. I., and Cole, B.: Spectrally consistent scattering, absorption, and polarization properties of atmospheric ice crystals at wavelengths from 0.2 to $100 \mu \mathrm{m}$, J. Atmos. Sci., 70, 330-347, https://doi.org/10.1175/JAS-D-12-039.1, 2013.

Yang, P., Liou, K.-N., Bi, L., Liu, C., Yi, B., and Baum, B. A.: On the radiative properties of ice clouds: light scattering, remote 
sensing, and radiation parameterization, Adv. Atmos. Sci., 32, 32-63, https://doi.org/10.1007/s00376-014-0011-z, 2015.

Yi, B., Yang, P., Baum, B. A., L'Ecuyer, T., Oreopoulos, L., Mlawer, E. J., Heymsfield, A. J., and Liou, K.-N.: Influence of ice particle surface roughening on the global cloud radiative effect, J. Atmos. Sci., 70, 2794-2807, https://doi.org/10.1175/JASD-13-020.1, 2013.
Zhang, Z:: A flexible new technique for camera calibration, IEEE T. Pattern Anal., 22, 1330-1334, 2000. 